\title{
Anthracycline and Trastuzumab-Based Therapy in Early Stage Breast Cancer: Do the Data Justify Cardiac Surveillance?
}

\author{
Natalie Berger ${ }^{1}$, Charles L. Shapiro ${ }^{2 *}$
}

'Hematology/Oncology Fellowship Program, Icahn School of Medicine at Mount Sinai, New York, NY

${ }^{2}$ Division of Hematology and Medical Oncology, Icahn School of Medicine at Mount Sinai, New York, NY

\section{Article Info}

\section{Article Notes}

Received: June 12, 2018

Accepted: July 24, 2018

\section{${ }^{\star}$ Correspondence:}

Dr. Charles L. Shapiro, MD, Professor of Medicine, Division of Hematology and Oncology, Icahn School of Medicine at Mount Sinai Uptown, One Gustave Levy Place, Box 1079, New York, NY 1023; Telephone No: 212-241-3131; Fax No: 212-241-40;

Email: charles.shapiro@mssm.edu.

C) 2018 Shapiro CL. This article is distributed under the terms of the Creative Commons Attribution 4.0 International License.

\section{Introduction}

Anthracycline-based and trastuzumab-containing regimens make a significant impact on reducing the risk of distant metastases and improving cause-specific and overall survival in early stage breast cancer. However, these regimens may cause cardiomyopathy during and after treatment. Despite anthracyclines being in use for nearly fifty years, there are no evidence-based guidelines for cardiac surveillance in asymptomatic breast cancer survivors. In fact, the current guidelines for cardiac monitoring while on trastuzumab therapy were from clinical trials, with empiricism and not data informing the recommendations for cardiac monitoring.

Surveillance recommendations vary depending on which guidelines one reads. The American Society of Clinical Oncology (ASCO) recommends no further cardiac imaging in asymptomatic women with early breast cancer who received cumulative doses (called for purposes of this review "limited doses") of doxorubicin of less than $250 \mathrm{mg} / \mathrm{m}^{2}$ (or epirubicin $300 \mathrm{mg} / \mathrm{m}^{2}$ ) 1,2 .

The National Comprehensive Cancer Network (NCCN) recommends a baseline echocardiogram (ECHO) and repeat ECHO in one year for those women with one or more risk cardiac risk factors, including age over 65 years, diabetes, hypertension, hyperlipidemia, family history of cardiomyopathy, atrial fibrillation, coronary artery disease, or structural heart disease ${ }^{3}$. In contrast, the European Society for Medical Oncology ${ }^{4}$, the Canadian Cardiovascular Society ${ }^{5}$, Italian Society of Cardiology and Working Group of Drug Cardiotoxicity and Cardioprotection ${ }^{6}$, the American Society of Echocardiography and European Association of Cardiovascular Imaging ${ }^{7}$, and others ${ }^{8,9}$ recommend routine cardiac surveillance alone or the use of biomarkers plus cardiac imaging for surveillance to detect early declines in left ventricular function. Furthermore, in some instances, some guidelines endorse the initiation of cardiac medications in asymptomatic women.

Conflicting guideline recommendations leave clinicians and women with early breast cancer unsure about what surveillance recommendations are best in asymptomatic women who received limited doses of anthracycline and trastuzumab-based regimens. Reviewed in this commentary is the lack of evidence to support cardiac surveillance in asymptomatic women with breast cancer receiving limited-dose exposure to anthracyclines. 


\section{Type I and Type II Cardiotoxicity}

It is essential to distinguish between anthracyclineinduced myocardial damage (Type I) and trastuzumabinduced cardiac dysfunction (Type II). Type I myocardial injury causes direct myocyte death as confirmed by endomyocardial biopsy studies and is irreversible ${ }^{10}$. The frequency of Type I is related to the total cumulative dose of anthracycline; the route of administration with intravenous bolus dosing causing higher rates of cardiomyopathy than weekly lower doses or continuous infusions; underlying cardiac disease; aging (over age 65 years); or a history of mediastinal radiation. In contrast, there are no risk factors for Type II, and it does not cause myocardial cell death ${ }^{10}$. Other features that distinguish Type II from Type I are that it is not dose-dependent, and holding trastuzumab for a period of four to six weeks and repeating cardiac imaging often results in left ventricular ejection function (LVEF) recovery with or without cardiac medications. When LVEF recovers, retreatment with trastuzumab-based regimens is possible in more than fifty percent of women ${ }^{11}$. Thus, Type I and Type II are very different regarding their pathogeneses, and clinical implications and outcomes.

\section{Lack of a Standard Definition of Cardiotoxicity by LVEF Declines}

Whereas the development of congestive heart failure (CHF) or cardiac death are easy to define endpoints, there is no standard definition of cardiotoxicity based on the LVEF declining. Generally, the definitions involve an asymptomatic decreases LVEF within the normal range and asymptomatic or symptomatic decreases in LVEF below the normal range. Varying studies define cardiotoxicity as either a fall in LVEF to less than $50 \%$, an asymptomatic reduction in LVEF of more than $10 \%$ to LVEF to less than $55 \%$, LVEF decrease more than $20 \%$ from baseline or an asymptomatic fall in LVEF of $10-15 \%$ within the normal range or some combination of these $\mathrm{e}^{12,13}$. These varying definitions unduly complicate cross-trial comparisons and impede the link between decreases in LVEF as a surrogate endpoint on the clinically meaningful endpoints of CHF or cardiac deaths.

The Scope of the Problem: Limited Doses of Anthracyclines and the Incidence of Clinically Meaningful Cardiac End Points

Table 1 describes the rates of CHF and cardiac deaths in

Table 1: The scope of the problem: Meaningful Cardiac Events with Limited Doses of Anthracyclines Alone or Anthracyclines followed by Trastuzumab

\begin{tabular}{|c|c|c|c|c|c|c|c|c|}
\hline Trial & $\mathbf{N}$ & $\begin{array}{c}\text { Follow-up } \\
(y r)\end{array}$ & $\begin{array}{c}\text { Total Median } \\
\text { Doxorubicin }\left(\mathrm{mg} / \mathrm{m}^{2}\right)\end{array}$ & $\begin{array}{c}\Delta \text { LVEF } \\
(\%)\end{array}$ & $\begin{array}{l}\text { CHF } \\
(\%)\end{array}$ & $\begin{array}{c}\text { Anthracyclines } \\
\text { HR or OR }(95 \% \mathrm{Cl})\end{array}$ & $\begin{array}{l}\text { No Anthracyclines } \\
\text { HR or OR }^{*}(95 \% \mathrm{Cl})\end{array}$ & $\begin{array}{c}\text { Cardiac Deaths } \\
\text { (\%) }\end{array}$ \\
\hline \multicolumn{9}{|l|}{ Anthracyclines only } \\
\hline \multicolumn{9}{|c|}{ Prospective randomized trials } \\
\hline \multirow[t]{2}{*}{ Shulman 2012 (14) } & 1107 & 5.3 & 240 & NA & 0.2 & NA & NA & 0.1 \\
\hline & 766 & 5.3 & 360 & NA & 0.7 & NA & NA & 0.1 \\
\hline Advani 2016 (15) & 664 & 6 & 240 & -3 & 0.6 & NA & NA & 0.3 \\
\hline \multicolumn{9}{|l|}{ Case-Control } \\
\hline $\begin{array}{l}\text { Thavendiranathan } \\
2016 \text { (17) }\end{array}$ & 10,160 & 3 & NA & NA & 3.0 & $\begin{array}{c}0.97 \\
(0.73 \text { to } 1.27)\end{array}$ & $\begin{array}{c}0.94 \\
(0.41 \text { to } 2.20)\end{array}$ & 0.5 \\
\hline \multicolumn{9}{|l|}{ SEER Registry } \\
\hline Doyle $2005^{\&}(19)$ & 5,571 & 5 & NA & NA & 3.0 & $\begin{array}{c}1.38 \\
(1.25 \text { to } 1.52)\end{array}$ & $\begin{array}{c}1.13^{\lambda} \\
(1.03 \text { to } 1.23)\end{array}$ & NA \\
\hline Pinder $2007^{\&}(20)$ & 8,083 & 10 & NA & NA & 3.0 & $\begin{array}{c}1.26 \\
(1.12 \text { to } 1.42)\end{array}$ & $\begin{array}{c}0.90^{\pi} \\
\text { (0.86 to } 0.99)\end{array}$ & NA \\
\hline \multicolumn{9}{|c|}{ Anthracyclines followed Trastuzumab } \\
\hline \multicolumn{9}{|c|}{ Prospective randomized trials } \\
\hline \multirow[t]{2}{*}{ Advani $2016(15)$} & 710 & 6 & 240 & -3 & $2.8^{\gamma}$ & NA & NA & 0.1 \\
\hline & 570 & 6 & 240 & -3 & $3.5^{f}$ & NA & NA & 0.2 \\
\hline \multirow[t]{2}{*}{ Cameron 2017 (21) } & 1697 & 11 & NA & NA & $1.0^{*}$ & NA & NA & NA \\
\hline & 1702 & 11 & NA & NA & $0.1^{*}$ & NA & NA & NA \\
\hline \multicolumn{9}{|l|}{ Case-Control } \\
\hline $\begin{array}{l}\text { Thavendiranathan } \\
2016 \text { (17) }\end{array}$ & 3,250 & 3 & NA & NA & 4.2 & $\begin{array}{c}3.96 \\
\text { (3.01 to } 5.22)\end{array}$ & $\begin{array}{c}0.81 \\
(0.25 \text { to } 2.66)\end{array}$ & 1.0 \\
\hline
\end{tabular}

Abbreviations: years (yr); left ventricular ejection fraction (LVEF); congestive heart failure (CHF); hazard ratio (HR); odds ratio (OR); not available (NA). "Adjusted hazard or odds ratio. ${ }^{\lambda}$ Women with early-stage breast cancer who received adjuvant non-anthracycline containing chemotherapy. ${ }^{\top}$ Women with early-stage breast cancer who received no adjuvant chemotherapy. ${ }^{\Upsilon}$ Sequentially or ${ }^{\mathrm{f}}$ concurrently administered paclitaxel and trastuzumab. "Major cardiac events in sequentially administered trastuzumab (1\%) and controls $(0.1 \%)$. 
recent randomized trials. The crude rates of $\mathrm{CHF}$ and cardiac deaths with anthracyclines alone are dose-dependent and vary from $0.2 \%$ to $0.7 \%$ and $0.1 \%$ to $0.3 \%$, respectively ${ }^{14,15}$. However, these are women who participated in randomized prospective trials, a group that is considered healthier than the general population ${ }^{16}$.

An extensive retrospective case-control study from Ontario Canada compared over 10,000 women with breast cancer who received anthracyclines to over 92,000 heathy women without breast cancer ${ }^{17}$. The median age of anthracycline-treated women was 52 years, 31\% had hypertension, $11 \%$ had diabetes, and the median Charleston co-morbidity index was $2^{18}$. The cumulative dose of doxorubicin was unknown. With a median follow-up of just over three years, in the cohort that received anthracyclines the rates of $\mathrm{CHF}$ that required hospitalization and cardiac deaths were $3 \%$ and $0.5 \%$, respectively. In the controls the corresponding rates of major cardiac event were $0.5 \%$ and cardiac deaths was $0.5 \%$. In a multivariate analysis adjusting for age (over 65 years), diabetes, radiation, and cancer stage for the primary outcome ( $\mathrm{CHF}$ that required hospitalization and cardiac deaths) was a hazard ratio (HR) of 0.97 (95\% CI 0.73 to 1.27) for the anthracycline group. Thus, in the multivariate analysis, there was no increase in major cardiac events for the group that received anthracyclines.

In contrast, there was the group of over 3,000 women who received in the same control study, sequentially administered trastuzumab. In this case-cohort there was nearly 4-fold higher HR for major cardiac events (CHF) compared to controls that did not receive anthracyclines (HR ratio of 3.96 (95\% CI 3.01 to 5.22). However, the adjusted death rates were not higher (HR ratio of 0.81 (95\% CI 0.25 to 2.66).

In another set of registry studies from the Surveillance Epidemiology and End Results (SEER) shows that when women with breast cancer age 65 years or older receive anthracyclines, there are slightly higher risks of developing CHF. In one study, women with breast cancer who received anthracyclines and those that did not receive chemotherapy (controls) the adjusted HR for CHF was 1.26 (95\%CI 1.12 to $1.42, \mathrm{p}<0.001$ ) and HR of $0.90,(95 \%$ CI 0.86 to 0.99 ), respectively. In another study of similar design, the adjusted odds ratio (OR) for CHF was 1.38 (95\% CI 1.25 to 1.52) and 1.13 (95\%CI 1.03 to 1.23 ) for the adjuvant chemotherapy containing doxorubicin and a non-anthracycline containing adjuvant chemotherapy, respectively (Table 1$)^{19,20}$.

Table 1 also describes anthracyclines followed by trastuzumab. First, it is important to understand the benefits of adding trastuzumab to standard anthracyclinecontaining chemotherapy regimens. In a combined analysis of two pivotal trials the hazard ratio for mortality was $0.67(95 \%$ CI 0.48 to $0.93, p=0.015)$ comparing doxorubicin (60 mg/m²) and cyclophosphamide $(600 \mathrm{mg} /$ $\mathrm{m}^{2}$ ) for 4 cycles every 2-weeks followed by paclitaxel (80 $\mathrm{mg} / \mathrm{m}^{2}$ ) for 12 weekly cycles with or without concurrent trastuzumab in over 3600 women with early-stage breast cancer ${ }^{13}$. Sequentially administration of trastuzumab, after all chemotherapy has ended, has less cardiac toxicity than concurrent administration of trastuzumab with paclitaxel, but sequential trastuzumab was less effective ${ }^{15,17,21}$. Thus, the preferred of administration of trastuzumab is concurrent with the paclitaxel.

In absolute terms, if 1000 HER2 over-expressing women received $\mathrm{AC}$ and taxanes without trastuzumab, 917 (91.7\%) would survive, 6 (0.6\%) would experience $\mathrm{CHF}$, and $3(0.3 \%)$ die from cardiac causes over a median of 5 years. When trastuzumab is added concurrently to anthracycline-taxane-based chemotherapy, 943 will survive, 35 (3.5\%) would experience CHF and 2 (0.2\%) die from cardiac causes ${ }^{13}$. The left ventricular dysfunction from trastuzumab improves in the majority of women with stopping the trastuzumab either with or without cardiac medications. Over $50 \%$ of these women can receive trastuzumab again without risks of cardiotoxicity ${ }^{15}$.

Thus, the benefits in lives saved far outweighs cardiac deaths. It is extremely rare to develop late anthracycline or trastuzumab related CHF or cardiac deaths with follow-up durations of nine to ten years ${ }^{15,22}$. Finally, in a very recent trial six months of trastuzumab was not inferior to 12 months, and the six-month treatment had less CHF and cardiac deaths ${ }^{23}$.

\section{Cardiac Imaging to Monitor for Cardiotoxicity}

The early detection of subclinical decreases in LVEF limits irreversible cardiac damage in Type 1 and may have a substantial impact on the reversibility of Type 2 cardiac dysfunction ${ }^{24}$. 2D echocardiography (ECHO) is the most common noninvasive imaging modality to monitor as it is easily performed and does not have any radiation exposure. A low baseline LVEF (i.e., 50\% to 55\%), asymptomatic declines in LVEF during treatment, and age older than 65 years increase the risks of CHF. However, 2D ECHO has poor sensitivity for the detection of subclinical myocardial damage ${ }^{9,25,26}$. Newer techniques such as real-time 3D ECHO, Doppler tissue imaging, and cardiac magnetic resonance imaging have higher sensitivity for detecting myocardial damage than 2D ECHO, but their higher costs and less availability limit the use of these newer techniques ${ }^{7}$.

The use of global longitudinal strain (GLS) which measures myocardial deformation as a percentage of change from the original dimension per unit time is a measurement on echocardiogram can identify wall motion abnormalities before detecting decreases in $\mathrm{LVEF}^{27}$. There are limited data to support the prognostic value of measuring $\mathrm{GLS}^{7}$. However, The European Society of 
Cardiology ${ }^{28}$ and others ${ }^{6,27}$ do not support the use of GLS alone to stop or change anthracyclines. Larger prospective studies are needed to determine if GLS can predict CHF.

The value of a baseline echocardiogram is called into question based on a retrospective study of 220 women by Mina et al. ${ }^{29}$ The population was typical of a cohort of women with early-stage breast cancer with a median age was 52 years old, $2 \%$ of women had coronary artery disease, $16 \%$ had diabetes, $24 \%$ had hypertension, 35\% were smokers, and $32 \%$ were obese (BMI >30). Fifteen (7\%) had a wall motion abnormality on echocardiogram, $6(3 \%)$ had LVEF of less than $50 \%$ and only in $3(1.3 \%)$ did chemotherapy regimen change based on these findings. Prospective studies are necessary to determine the value of the baseline echocardiogram.

\section{Cardiac Biomarkers}

Cardiac biomarkers include Troponin I, B-type natriuretic peptide (BNP), and N-terminal B-type natriuretic peptide (NT-proBNP). Troponin-I has many advantages including high specificity for cardiac tissue, high sensitivity for detecting myocyte necrosis, low cost, and is minimally invasive. Cardinale et al. did the most extensive study to examine cardiac troponins to detect early chemotherapy-induced cardiotoxicity. These were individuals receiving anthracyclines before high-dose chemotherapy with autologous or allogeneic bone marrow transplant ${ }^{30}$. Whereas troponins sequentially collected were able to stratify high or low-risk groups for risks of $\mathrm{CHF}$, these individuals were very different from women who receive limited doses of anthracyclines.

Also, in patients with early-stage HER2-positive breast cancer receiving anthracyclines and HER2-targeted therapies (trastuzumab or lapatinib), troponin I as an early indicator of cardiotoxicity did not predict for $\mathrm{CHF}^{31,32}$. Likewise conflicting data use of BNP and N-terminal BNP ${ }^{32-}$ ${ }^{34}$ as a predictive marker for CHF.

The Value of Repeated Cardiac Imaging in Women with Breast Cancer Receiving Limited Doses of Anthracyclines

Whereas many cardio-oncology and cardio-imaging organizations recommend subsequent cardiac imaging with or without cardiac biomarkers in asymptomatic women who received limited doses of anthracyclines ${ }^{4-9}$, there are little data to support these recommendations. All of these recommendations are consensus-based and lack high-quality level I evidence that subsequent cardiac imaging will prevent $\mathrm{CHF}$ or cardiac deaths.

Are there harms to doing repeat cardiac imaging with or without cardiac biomarkers or treatment of surrogate endpoints (i.e., GLS, troponins, BNP) with cardiac medications in asymptomatic women? ${ }^{24}$ First, the frequency of CHF or cardiac deaths is very low (Table 1 ). Second, these surrogate endpoints lack validation for $\mathrm{CHF}$ and cardiac deaths in women with limited exposure to anthracyclines. Third, giving a diagnosis of a "cardiac problem" can cause distress and impact negatively on health-related quality of life, and fourth is the costs of surveillance testing when the value of that care (i.e., the prevention $\mathrm{CHF}$ or cardiac deaths/over the total costs, including the distress and negative impact on healthrelated quality of life of surveillance testing) is unknown.

\section{What to do with Women Who are Going to Receive Limited Doses of Anthracyclines?}

A past medical history of hypertension, diabetes, strong family history or personal history of cardiac disease, is of older age (greater than 65 years old) or has a low baseline LVEF (50-55\%) merits consideration of substituting a non-anthracycline-based regimen. All the anthracyclinebased chemotherapy regimens have corresponding nonanthracycline-based regimens. If a woman develops fatigue, shortness of breath, nocturnal dyspnea, or lower extremity edema during or after receiving limiteddose anthracyclines, perform a workup that includes the cardiorespiratory systems as clinically indicated. If significant decreases in LVEF or CHF occurs, then cardiology or cardio-oncology referral is indicated. We concur with recent Breast Cancer ASCO Survivorship Guidelines ${ }^{1}$, and the recent ASCO Clinical Practice Guidelines on the Prevention and Monitoring of Cardiac Dysfunction in Adult Survivors ${ }^{2}$. No cardiac surveillance is medically warranted with the currently availability tools for predicting risk and monitoring cardiac function.

\section{Acknowledgements}

CLS was responsible for conceptualization of article. NB was responsible for generating the first draft. CLS was responsible for multiple edits of first draft and creating Table 1. CLS and NB both read the final manuscript before submission.

\section{References}

1. Runowicz CD, Leach CR, Henry NL, et al. American Cancer Society/ American Society of Clinical Oncology Breast Cancer Survivorship Care Guideline. J Clin Oncol. 2016; 34(6): 611-35.

2. Armenian SH, Lacchetti C, Barac A, et al. Prevention and Monitoring of Cardiac Dysfunction in Survivors of Adult Cancers: American Society of Clinical Oncology Clinical Practice Guideline. J Clin Oncol. 2017; 35(8): 893-911.

3. NCCN Guideline Version 2.2015 NCCN Guideline for Anthracyclineinduced Cardiac Toxicity 2015.

4. Curigliano G, Cardinale D, Suter T, et al. Cardiovascular toxicity induced by chemotherapy, targeted agents and radiotherapy: ESMO Clinical Practice Guidelines. Ann Oncol. 2012; 23 Suppl 7: vii155-66.

5. Virani SA, Dent S, Brezden-Masley C, et al. Canadian Cardiovascular Society Guidelines for Evaluation and Management of Cardiovascular Complications of Cancer Therapy. Can J Cardiol. 2016; 32(7): 831-41. 
6. Maurea N, Spallarossa P, Cadeddu C, et al. A recommended practical approach to the management of target therapy and angiogenesis inhibitors cardiotoxicity: an opinion paper of the working group on drug cardiotoxicity and cardioprotection, Italian Society of Cardiology. J Cardiovasc Med (Hagerstown). 2016; 17 Suppl 1: S93-S104.

7. Plana JC, Galderisi M, Barac A, et al. Expert consensus for multimodality imaging evaluation of adult patients during and after cancer therapy: a report from the American Society of Echocardiography and the European Association of Cardiovascular Imaging. Eur Heart J Cardiovasc Imaging. 2014; 15(10): 1063-93.

8. Schmitz KH, Prosnitz RG, Schwartz AL, et al. Prospective surveillance and management of cardiac toxicity and health in breast cancer survivors. Cancer. 2012; 118(8 Suppl): 2270-6.

9. Zagar TM, Cardinale DM, Marks LB. Breast cancer therapy-associated cardiovascular disease. Nat Rev Clin Oncol. 2016; 13(3): 172-84.

10. Ewer MS, Ewer SM. Cardiotoxicity of anticancer treatments. Nat Rev Cardiol. 2015.

11. Perez EA, Suman VJ, Davidson NE, et al. Cardiac safety analysis of doxorubicin and cyclophosphamide followed by paclitaxel with or without trastuzumab in the North Central Cancer Treatment Group N9831 adjuvant breast cancer trial. J Clin Oncol. 2008; 26(8): 1231-8.

12. Tan-Chiu E, Yothers G, Romond E, et al. Assessment of cardiac dysfunction in a randomized trial comparing doxorubicin and cyclophosphamide followed by paclitaxel, with or without trastuzumab as adjuvant therapy in node-positive, human epidermal growth factor receptor 2-overexpressing breast cancer: NSABP B-31. J Clin Oncol. 2005; 23(31): 7811-9.

13. Romond EH, Perez EA, Bryant J, et al. Trastuzumab plus adjuvant chemotherapy for operable HER2-positive breast cancer. N Engl J Med. 2005; 353(16): 1673-84.

14. Shulman LN, Cirrincione CT, Berry DA, et al. Six cycles of doxorubicin and cyclophosphamide or Paclitaxel are not superior to four cycles as adjuvant chemotherapy for breast cancer in women with zero to three positive axillary nodes: Cancer and Leukemia Group B 40101. J Clin Oncol. 2012; 30(33): 4071-6.

15. Advani PP, Ballman KV, Dockter TJ, et al. Long-Term Cardiac Safety Analysis of NCCTG N9831 (Alliance) Adjuvant Trastuzumab Trial. J Clin Oncol. 2016; 34(6): 581-7.

16. Mailankody S, Prasad V. Overall Survival in Cancer Drug Trials as a New Surrogate End Point for Overall Survival in the Real World. JAMA Oncol. 2017; 3(7): 889-90.

17. Thavendiranathan $\mathrm{P}$, Abdel-Qadir H, Fischer HD, et al. Breast Cancer Therapy-Related Cardiac Dysfunction in Adult Women Treated in Routine Clinical Practice: A Population-Based Cohort Study. J Clin Oncol. 2016; 34(19): 2239-46.

18. Sundararajan V, Henderson T, Perry C, et al. New ICD-10 version of the Charlson comorbidity index predicted in-hospital mortality. J Clin Epidemiol. 2004; 57(12): 1288-94.

19. Doyle JJ, Neugut AI, Jacobson JS, et al. Chemotherapy and cardiotoxicity in older breast cancer patients: a population-based study. J Clin Oncol. 2005; 23(34): 8597-605.

20. Pinder MC, Duan Z, Goodwin JS, et al. Congestive heart failure in older women treated with adjuvant anthracycline chemotherapy for breast cancer. J Clin Oncol. 2007; 25(25): 3808-15.

21. Cameron D, Piccart-Gebhart MJ, Gelber RD, et al. 11 years' follow-up of trastuzumab after adjuvant chemotherapy in HER2-positive early breast cancer: final analysis of the HERceptin Adjuvant (HERA) trial. Lancet. 2017; 389(10075): 1195-205.

22. Ganz PA, Romond EH, Cecchini RS, et al. Long-Term Follow-Up of Cardiac Function and Quality of Life for Patients in NSABP Protocol B-31/NRG Oncology: A Randomized Trial Comparing the Safety and Efficacy of Doxorubicin and Cyclophosphamide (AC) Followed by Paclitaxel With AC Followed by Paclitaxel and Trastuzumab in Patients With Node-Positive Breast Cancer With Tumors Overexpressing Human Epidermal Growth Factor Receptor 2. J Clin Oncol. 2017; 35(35): 3942-8.

23. Earl H, Hiller, L, Vallier, A. et al. PERESPHONE Trial Investigators: 6 versus 12 months (m) adjuvant trastuzumab in patients (pts) with HER positive (+) early-breast cancer (EBC). Randomized phase 3 noninferiority trial with definite 4-year (yr) disease-free survival (DFS) results. J Clin Oncol. 2018; 38 (suppl ;506 abstr.).

24. Levis BE, Binkley PF, Shapiro CL. Cardiotoxic effects of anthracyclinebased therapy: what is the evidence and what are the potential harms. Lancet Oncol. 2017; 18(8): e445-e56.

25. Jensen BV, Skovsgaard T, Nielsen SL. Functional monitoring of anthracycline cardiotoxicity: a prospective, blinded, long-term observational study of outcome in 120 patients. Ann Oncol. 2002; 13(5): 699-709.

26. Ewer MS, Lenihan DJ. Left ventricular ejection fraction and cardiotoxicity: is our ear really to the ground. J Clin Oncol. 2008; 26(8): 1201-3.

27. Thavendiranathan P, Poulin F, Lim KD, et al. Use of myocardial strain imaging by echocardiography for the early detection of cardiotoxicity in patients during and after cancer chemotherapy: a systematic review. J Am Coll Cardiol. 2014; 63(25 Pt A): 2751-68.

28. Zamorano JL, Lancellotti P, Rodriguez Munoz D, et al. 2016 ESC Position Paper on cancer treatments and cardiovascular toxicity developed under the auspices of the ESC Committee for Practice Guidelines: The Task Force for cancer treatments and cardiovascular toxicity of the European Society of Cardiology (ESC). Eur Heart J. 2016; 37(36): 2768-801.

29. Mina A, Rafei H, Khalil M, et al. Role of baseline echocardiography prior to initiation of anthracycline-based chemotherapy in breast cancer patients. BMC Cancer. 2015; 15: 10.

30. Cardinale D, Sandri MT, Colombo A, et al. Prognostic value of troponin I in cardiac risk stratification of cancer patients undergoing high-dose chemotherapy. Circulation. 2004; 109(22): 2749-54.

31. Morris PG, Chen C, Steingart R, et al. Troponin I and C-reactive protein are commonly detected in patients with breast cancer treated with dose-dense chemotherapy incorporating trastuzumab and lapatinib. Clin Cancer Res. 2011; 17(10): 3490-9.

32. Fallah-Rad N, Walker JR, Wassef A, et al. The utility of cardiac biomarkers, tissue velocity and strain imaging, and cardiac magnetic resonance imaging in predicting early left ventricular dysfunction in patients with human epidermal growth factor receptor II-positive breast cancer treated with adjuvant trastuzumab therapy. J Am Coll Cardiol. 2011; 57(22): 2263-70.

33. Skovgaard D, Hasbak P, Kjaer A. BNP predicts chemotherapy-related cardiotoxicity and death: comparison with gated equilibrium radionuclide ventriculography. PLoS One. 2014; 9(5): e96736.

34. Ky B, Putt M, Sawaya H, et al. Early increases in multiple biomarkers predict subsequent cardiotoxicity in patients with breast cancer treated with doxorubicin, taxanes, and trastuzumab. J Am Coll Cardiol. 2014; 63(8): 809-16 